\title{
Aujeszky's disease and the effects of infection on Japanese swine herd productivity: a cross-sectional study
}

\author{
Itsuro YAMANE ${ }^{1) *}$, Sayoko ISHIZEKI ${ }^{2)}$ and Hisanori YAMAZAKI ${ }^{1)}$ \\ 1)Viral Disease and Epidemiology Research Division, National Institute of Animal Health, 3-1-5 Kannondai, Tsukuba, Ibaraki 305-0856, \\ Japan \\ ${ }^{2)}$ Summit Veterinary Services, 1276-2 Motogimachi, Maebashi, Gunma 371-0232, Japan
}

(Received 29 July 2014/Accepted 17 December 2014/Published online in J-STAGE 7 January 2015)

ABSTRACT. Pseudorabies virus (PRV) is endemic in some regions of Japan. We investigated the effects of PRV infection status on herd productivity. Serum samples were obtained from 48 swine herds in Japan. Within each herd, three serum samples were obtained from growing pigs at four different ages, as well as from sows in low and high parity groups. Sera were tested for antibodies against wild-type PRV via competitive ELISA. Herds were classified into PRV positive and negative groups based on serological results. Herds infected with PRV exhibited postweaning mortalities $(6.84 \%)$ that were significantly $(P=0.0018)$ higher than those in unaffected herds $(4.73 \%)$. Because of the reduced productivity in PRV positive herds, the current PRV eradication program must be strengthened.

KEY WORDS: Aujeszky's disease, PigINFO, postweaning mortality, pseudorabies virus, swine

doi: 10.1292/jvms.14-0385; J. Vet. Med. Sci. 77(5): 579-582, 2015

Infection with pseudorabies virus (PRV), the causative agent of Aujeszky's disease (AD), results in the deaths of young piglets through disorders of the central nervous system. In older infected pigs, respiratory syndromes are seen, while infected sows can experience abortion [4]. PRV has caused serious economic losses for the swine industry worldwide, and programs to eradicate PRV have been implemented in many countries [8, 10]. Within EU countries, these PRV eradication programs have been implemented to facilitate the free trade of pigs [2, 15]. In Japan, the first case of $\mathrm{AD}$ in piglets was reported in 1981 [5], with infections spreading to many different prefectures [16]. The Japanese Ministry of Agriculture, Forestry and Fisheries established prevention and control measures against PRV in 1991, with a regional eradication program implemented [9]. Successful eradication of PRV was completed in some areas, but there remained some regions of Japan where PRV was endemic. A new eradication campaign, based on successful eradication strategies conducted by other countries, commenced in 2009 [9]. For this campaign, all swine production areas were designated as one of 5 stages: I, preparation; IIa, enforcement of complete vaccination; IIb, transition phase; III, surveillance with serological testing and slaughter of seropositive animals; and IV, eradication completed. By March 2014, 36 of 47 prefectures were classified as stage IV. However, for the remaining 11 prefectures, 320 areas were classified as stage IIa or IIb, and 86 areas were classified as stage III, demonstrating that PRV remains endemic in some areas of

*Correspondence to: Yamane, I., National Institute of Animal Health, 3-1-5 Kannnonndai, Tsukuba-shi, Ibaraki 305-0856, Japan. e-mail: iyamane@affrc.go.jp

(C2015 The Japanese Society of Veterinary Science

This is an open-access article distributed under the terms of the Creative Commons Attribution Non-Commercial No Derivatives (by-nc-nd) License $<$ http://creativecommons.org/licenses/by-nc-nd/3.0/>.
Japan [7].

Although PRV-infected farms exist, the effects of PRV infection on swine productivity are unclear. Our study objective was to investigate the effects of PRV infection on herd productivity using a data recording system (PigINFO) recently developed for Japanese pig producers through a collaboration between the National Institute of Animal Health (NIAH) and the Japan Association of Swine Veterinarians (JASV) [17].

The study period was from 1 January to 31 December in 2011. Targeted herds were selected from clients of veterinarians that were members of the JASV who volunteered to be part of this study. Only farrow-to-finish herds were included, while herds that practiced piglet importation or exportation with other farms were excluded. Study herds were selected from prefectures where PRV was not eradicated by the beginning of 2011. The purpose of the study and its design were explained to participating herd owners by veterinarians. A total of 48 herds were included in the study, from the Aomori (2), Fukushima (4), Tochigi (4), Gumma (15), Saitama (2), Chiba (13), Kanagawa (6) and Kagoshima (2) prefectures.

From each of the herds, growing pigs were divided into 4 age groups $(60,90,120$ and 150 day-old), and three serum samples were randomly taken from growing pigs in each of these groups and from sows in low and high parity groups. Blood sampling was conducted twice during the study period. The obtained sera were tested for antibodies against wild-type PRV glycoprotein I (gI) using a commercially available competitive ELISA (IDEXX Laboratories, Inc., Westbrook, ME, U.S.A.). Herds were defined as positive, if any of the serum samples were positive. Herds were defined as negative, if all sera were negative for anti-gI antibodies.

Annual production data for 2011 were obtained from the PigINFO system [17] (Table 1) and used to calculate annual productivity parameters based on the definitions described in Table 2. Investigators were not informed of the names of 
Table 1. Annual herd production data for 2011

\begin{tabular}{ll}
\hline \multicolumn{1}{c}{ Variables } & \multicolumn{1}{c}{ Definition } \\
\hline Average female inventory (AFI) & Includes sows and mated, but not maiden, gilts (numbers were counted monthly; \\
& AFI was defined as the mean of the 12-month values) \\
Total number of marketed pigs (TNMP) & Total number of fattening pigs sold (excluding sows and boars sold) \\
Total number of pigs weaned (TNPW) & Total number of pigs weaned \\
Total number of pigs mated (TNM) & Total number of pigs mated \\
Total number of litters (TNL) & Total number of litters (excluding abortions) \\
Total number of pigs born alive (TNPB) & Total number of pigs born alive at farrowing (excluding stillborn and mummified piglets) \\
Total number of deaths at postweaning stage (TND) & Total number of deaths (including euthanized) at the post weaning stage \\
\hline
\end{tabular}

a) For farms using on-site pigs as replacement sows, data adjustments were carried out for TNMP based on the assumption that replacements were sold to the slaughterhouse.

Table 2. Definitions for annual productivity parameters

\begin{tabular}{ll}
\hline \multicolumn{1}{c}{ Variables } & \multicolumn{1}{c}{ Definition } \\
\hline Marketed pigs per sow (MP) & TNMP / AFI \\
Pigs weaned per mated female per year (PWMFY) & TNPW / AFI \\
Pigs weaned per litter (PWL) & TNPW / TNL \\
Litters per mated female per year (LMFY) & TNL / AFI \\
Pigs born alive per litter (PBA) & TNPB / TNL \\
Farrowing proportion (\%) (FP) & TNL / TNM \\
Post weaning mortality, \% (POWM) & TND / TNPW \\
Pre weaning mortality, \% (PRWM) & 1-TNPW / TNPB \\
\hline
\end{tabular}

individual herd owners to maintain confidentiality. Data handling was conducted based on a research contract between NIAH and JASV.

Productivity parameters in Table 2 were initially analyzed for normality of distributions by visually inspecting histograms and using the Shapiro-Wilk test. Log transformations were performed for variables that were not normally distributed and re-examined for their normality. For variables, such as AFI and postweaning mortality (POWM), log-transformed data were normally distributed and used for subsequent analyses. The mean productivity parameters between PRV-positive and -negative herds were compared using Student's t-test or the Mann-Whitney U-test depending on the normality of the parameters. Production parameters that were different $(P<0.1)$ compared with PRV status by univariate analysis were used as response variables for multiple regression analyses. PRV status and AFI were used as explanatory variables. All statistical analyses were performed using $\mathrm{R}$ version 2.13.0 ( $\mathrm{R}$ foundation for statistical computing 2011).

Antibodies against PRV were identified in 25.0\% (12/48) of herds (95\%CI: $13.6-39.6 \%$ ). Herds that were PRV-positive had significantly higher POWM compared with PRVnegative herds (Table 3). Using multiple regression analysis, PRV-positive herds had significantly higher POWM and lower marketed pigs (MP), litters per mated female per year (LMFY) and farrowing proportion (FP) than negative herds (Table 4).

The major clinical signs of acute phase PRV infections are increased piglet mortality with central nervous system disorders [1]. However, in the present study, PRV-positive herds did not have increased preweaning mortality. This is likely because all PRV-positive herds were using vaccines against PRV, and therefore, piglets within PRV-positive herds did not show clinical signs.

Potential confounding effects, such as farm size and productivity levels, were attempted to be excluded while investigating the association between PRV status and productivity. To achieve this goal, productivity parameters that had association $(P<0.1)$ with PRV status in the univariate analysis were used as respondent variables, and PRV status and AFI were included as explanatory variables to examine the association between PRV status, and productivity under controlling potential confounding effects by herd size. PRVpositive herds had significantly increased POWM compared to PRV-negative herds; this association was also supported by multiple regression analysis. Although the mean pigs weaned per mated female per year was not significantly different between PRV-negative and positive herds, PRVpositive herds had significantly lower MP, mainly due to increased POWM in PRV positive herds. Our results agree with those from a previous study, in which nursery and finishing pig mortalities increased during an $\mathrm{AD}$ epidemic [1]. However, this study demonstrated that PRV-infected herds had increased POWM even in the absence of AD epidemic. Animals that were seropositive for PRV were significantly more susceptible to becoming seropositive for Actinobacillus pleuropneumoniae serotype 2 and vice versa [3]. Clinical signs of experimental infection with A. pleuropneumoniae [12] or Mycoplasma hyopneumoniae [13] were exacerbated, if animals were co-infected with PRV. Using serological testing, we found that more than $80 \%$ of herds in this study were infected with $A$. pleuropneumoniae serotype 2 or 5 , and greater than $95 \%$ of herds were infected with 
Table 3. Mean productivity parameters and their association with PRV status

\begin{tabular}{|c|c|c|c|c|c|c|c|}
\hline \multirow{3}{*}{ Production parameter } & \multicolumn{6}{|c|}{ PRV status } & \multirow{3}{*}{$P$-value } \\
\hline & \multicolumn{3}{|c|}{ Negative } & \multicolumn{3}{|c|}{ Positive } & \\
\hline & $\mathrm{n}$ & mean & SD & $\mathrm{n}$ & mean & SD & \\
\hline Average Female Inventory a) & 36 & $\begin{array}{c}654.0 \\
(314.6)^{\mathrm{b})}\end{array}$ & 888.8 & 12 & $\begin{array}{c}1,007.7 \\
(331.3)^{\mathrm{b})}\end{array}$ & $1,639.7$ & 0.346 \\
\hline Marketed pigs per sow & 36 & 21.7 & 2.69 & 12 & 20.1 & 2.06 & 0.061 \\
\hline Pigs weaned per mated female per year & 36 & 23.3 & 2.35 & 12 & 22.3 & 1.99 & 0.229 \\
\hline Pigs weaned per litter & 36 & 9.88 & 0.74 & 12 & 9.80 & 0.41 & 0.647 \\
\hline Litters per mated female per year ${ }^{c)}$ & 36 & 2.35 & 0.12 & 12 & 2.28 & 0.15 & 0.083 \\
\hline Pigs born alive per litter & 36 & 10.91 & 0.79 & 12 & 10.86 & 0.50 & 0.850 \\
\hline Farrowing proportion (\%) & 36 & 85.1 & 7.40 & 12 & 80.7 & 6.39 & 0.058 \\
\hline Post weaning mortality $(\%)^{a)}$ & 36 & 4.73 & 1.78 & 12 & 6.84 & 2.29 & 0.0018 \\
\hline Pre weaning mortality (\%) & 36 & 9.42 & 3.11 & 12 & 9.72 & 3.02 & 0.766 \\
\hline
\end{tabular}

a) Original data were not normally distributed, while log-transformed data were used for Student's $t$-test. b) Data in parentheses were median values for this group. c) Original data and log-transformed data were not normally distributed. The Mann-Whitney test was applied.

Table 4. Effects of PRV status on 4 different production parameters selected from univariate analysis

\begin{tabular}{llrcrc}
\hline \multicolumn{1}{c}{ Response variables } & Explanatory variables & \multicolumn{1}{c}{$\beta$} & Standard error of $\beta$ & t-value & $P$ \\
\hline Marketed pigs per sow & Intercept & 17.007 & 1.946 & 8.740 & 0.000 \\
& PRV status & -1.818 & 0.818 & -2.223 & 0.031 \\
& AFI & 0.810 & 0.323 & 2.505 & 0.016 \\
\hline \multirow{2}{*}{ Litters per mated female per year } & Intercept & 2.028 & 0.089 & 22.823 & 0.000 \\
& PRV status & -0.086 & 0.037 & -2.306 & 0.026 \\
& AFI & 0.055 & 0.015 & 3.741 & 0.001 \\
\hline Farrowing proportion & Intercept & 0.668 & 0.050 & 13.233 & 0.000 \\
& PRV status & -0.051 & 0.021 & -2.393 & 0.021 \\
& AFI & 0.031 & 0.008 & 3.716 & 0.001 \\
\hline Post weaning mortality & Intercept & 0.010 & 0.014 & 0.688 & 0.495 \\
& PRV status & 0.020 & 0.006 & 3.291 & 0.002 \\
& AFI & 0.006 & 0.002 & 2.684 & 0.002 \\
\hline
\end{tabular}

M. hyopneumoniae(data not shown). Co-infection with these respiratory pathogens and PRV might have caused sporadic pneumonia at the postweaning stage, resulting in increased POWM in PRV-positive herds. Reduced FP and LMFY were observed in PRV-positive herds, which agrees with previous reports in which PRV infection can be the cause of reproductive failure, including abortion [4]. However, the actual association between PRV-status and reproductive performance needs to be further examined, as $P$ values for these parameters were marginal in this study.

We did not observe a significant difference in the AFI of ADV-positive or -negative herds. Reports concerning the influence of herd size on PRV seroprevalence are conflicting. Some researchers have reported no association between PRV status and herd size [11], which supports our findings in the current study. Others have suggested an increased risk of PRV infection with larger herd sizes [2, 6]. Conflicting results among studies could be the result of confounding factors, such as stocking density and management procedures, including biosecurity measures [14]. Targeted herds in this study were not representative of all Japanese swine farms, and volunteer bias for participation might have existed for both veterinarians and herd owners. This bias could have resulted in the inclusion of herds with higher levels of animal hygiene and those with motivated owners.

Higher POWM in PRV positive herds might have contributed to reduced MP, which subsequently resulted in significant economic losses for farmers. Other losses due to the cost of disease control and eradication and trade restriction have been estimated [4]. Producers, veterinarians and all levels of government need to collaborate to successfully eradicate PRV from Japan.

ACKNOWLEDGMENTS. This study was supported by collaborative research grants from the NIAH and JASV. We thank the veterinarians and farmers who participated in this study.

\section{REFERENCES}

1. Bech-Nielsen, S., Miller, G. Y., Bowman, G. L., Dodaro, S. J. and Orloski-Snider, K. A. 1992. Economic impact of an epizootic of pseudorabies in a commercial swine herd in Ohio, achieving test negative status and quarantine release by use of vaccination 
and test and removal. J. Am. Vet. Med. Assoc. 200: 1817-1823. [Medline]

2. Boelaert, F., Deluyker, H., Maes, D., Godfroid, J., Raskin, A., Varewijck, H., Pensaert, M., Nauwynck, H., Castryck, F., Miry, C., Robijns, J. M., Hoet, B., Segers, E., Van Vlaenderen, I., Robert, A. and Koenen, F. 1999. Prevalence of herds with young sows seropositive to pseudorabies (Aujeszky's disease) in northern Belgium. Prev. Vet. Med. 41: 239-255. [Medline] [CrossRef]

3. Elbers, A. R., Tielen, M. J., Cromwijk, W. A. and Hunneman, W. A. 1990. Sero-epidemiological screening of pig sera collected at the slaughterhouse to detect herds infected with Aujeszky's disease virus, porcine influenza virus and Actinobacillus (Haemophilus) pleuropneumoniae in the framework of an integrated quality control (IQC) system. Vet. Q. 12: 221-230. [Medline] [CrossRef]

4. Inch, C. 1998. An overview of pseudorabies (Aujeszky's disease) and vesicular stomatitis from the Canadian Animal Health Network. Can. Vet. J. 39: 23-32. [Medline]

5. Itakura, C., Nakatsuka, J. and Goto, M. 1981. An incidence of pseudorabies (Aujeszky's Disease) in piglets in Japan. Jpn. J. Vet. Sci. 43: 923-927. [Medline] [CrossRef]

6. Leontides, L., Ewald, C., Mortensen, S. and Willeberg, P. 1995. Factors Associated with the Seroprevalence of Aujeszky's Disease Virus in Seropositive Breeding Herds of Northern Germany during Area-Wide Compulsory Vaccination. Prev. Vet. Med. 23: 73-85. [CrossRef]

7. Ministry of Agriculture, Forestry and Fisheries, 2014. [cited 2014 November 3]. Available from http://www.maff.go.jp/j/ syouan/douei/eisei/e_ad/pdf/ad_status_map_141006.pdf.

8. Müller, T., Batza, H. J., Schluter, H., Conraths, F. J. and Mettenleiter, T. C. 2003. Eradication of Aujeszky's disease in Germany. J. Vet. Med. B Infect. Dis. Vet. Public Health 50: 207-213. [Medline] [CrossRef]
9. Ogikubo, T. 2009. Revision of points for eradication of the Aujeskey's disease. All About Swine 34: 3-6 (in Japanese).

10. Pensaert, M. and Morrison, R. B. 2000. Challenges of the final stages of the ADV eradication program. Vet. Res. 31: 141-145. [Medline]

11. Rodríguez-Buenfil, J. C., Alvarez-Fleites, M., Alzina-Lopez, A., Arjona-Torres, M. G., Segura-Correa, J. C. and Villegas-Perez, S. 2002. Risk factors for Aujeszky's disease in pig herds and detection of field virus antibodies in fattening pigs in the state of Yucatan, Mexico. Prev. Vet. Med. 53: 205-213. [Medline] [CrossRef]

12. Sakano, T., Shibata, I., Samegai, Y., Taneda, A., Okada, M., Irisawa, T. and Sato, S. 1993. Experimental pneumonia of pigs infected with Aujeszky's disease virus and Actinobacillus pleuropneumoniae. J. Vet. Med. Sci. 55: 575-579. [Medline] [CrossRef]

13. Shibata, I., Okada, M., Urono, K., Samegai, Y., Ono, M., Sakano, T. and Sato, S. 1998. Experimental dual infection of cesarean-derived, colostrum-deprived pigs with Mycoplasma hyopneumoniae and pseudorabies virus. J. Vet. Med. Sci. 60: 295-300. [Medline] [CrossRef]

14. Stegeman, A. 1995. Pseudorabies virus eradication by area-wide vaccination is feasible. Vet. Q. 17: 150-156. [Medline] [CrossRef]

15. Tamba, M., Calabrese, R., Finelli, E. and Cordioli, P. 2002. Risk factors for Aujeszky's-disease seropositivity of swine herds of a region of northern Italy. Prev. Vet. Med. 54: 203-212. [Medline] [CrossRef]

16. Yamada, S. 2008. Strategy of control for Aujeszky's disease. Proc. Jpn. Pig Vet. Soc. 52: 22-28 (in Japanese).

17. Yamane, I., Ishizeki, S. and Yamazaki, H. 2013. A survey of profitability and productivity variables and their component analysis in farrow-to-finish swine farms in Japan. J. Vet. Epidemiol. 17: 44-51. [CrossRef] 